OPEN ACCESS

Edited and reviewed by: Alessandro Rossi, Nello Carrara Institute of Applied Physics (IFAC), Italy

*Correspondence: Guglielmo S. Aglietti g.aglietti@auckland.ac.nz

Specialty section:

This article was submitted to Space Debris,

a section of the journal

Frontiers in Space Technologies

Received: 08 May 2020 Accepted: 19 May 2020

Published: 16 June 2020

Citation:

Aglietti GS (2020) From Space Debris to NEO, Some of the Major Challenges for the Space Sector.

Front. Space Technol. 1:2. doi: 10.3389/frspt.2020.00002

\section{From Space Debris to NEO, Some of the Major Challenges for the Space Sector}

\author{
Guglielmo S. Aglietti* \\ Department of Mechanical Engineering, The University of Auckland, Auckland, New Zealand
}

Keywords: space debris, active debris removal (ADR), planetary protection, in-orbit servicing, space traffic management (STM), space surveillance and tracking, space situational awareness (SSA)

\section{INTRODUCTION}

Society's reliance on space assets has grown to the point that today these are part of every modern country's infrastructure. Services provided thanks to space technologies such as for example, Global Navigation Satellite Systems have become critical (Hesse and Hornung, 2015) for smooth operations in a variety of sectors, from telecommunications to transport to banking, and the list could continue.

Even the general public has become accustomed to using satellite services like satellite television or the satnav on mobile phones. Hence, any threat to our space assets is a very significant issue for society.

As of February 2020, there were about 5,500 satellites in space ${ }^{1}$ but only about 2,300 were actually functioning, which means about 3,200 defunct satellites are still orbiting Earth, together with upper stages and fairings of rockets and a variety of smaller objects produced by break-ups, explosions, collisions, degradation or other anomalous events that resulted in the production of fragments. Under the collective name of space debris, these objects have a size distribution that ranges from large intact bodies (e.g., parts of rockets or large satellites with a size larger than $10 \mathrm{~m}$ and weight of several tons) down to millimeter-sized fragments like scales of paint or solidified droplets of coolant. Early 2020 estimates showed that there were 34,000 objects larger than $10 \mathrm{~cm}$, 900,000 objects from $>1$ to $10 \mathrm{~cm}$, and a staggering 128 million objects from $>1 \mathrm{~mm}$ to $1 \mathrm{~cm}$.

Given their high velocity and consequent high kinetic energy, even small pieces of debris pose a significant threat to operating satellites, as they could hit them with catastrophic consequences and the loss of potentially critical services.

At the same time, high energy collisions between larger bodies can produce real explosions that can create thousands of fragments. These, in turn, can collide with other orbiting objects, triggering a chain reaction and a snowball effect that could render whole orbits unusable. This extreme scenario (Kessler Syndrome), initially studied by Kessler in the '70s (Kessler and Cour-Palais, 1978), is not far from reality, as a handful of collisions have already happened. Perhaps the most famous is the one between Russian military communications satellite Cosmos 2,251 and a satellite of the Iridium constellation (Wang, 2010), which produced a step increase in the debris population.

With more satellite applications currently being developed that demand a growing number of satellites (e.g., constellations of hundreds of satellites are being deployed to provide worldwide connectivity or a World Wide Web), the issue of space debris is becoming more significant (Virgili et al., 2016).

\footnotetext{
${ }^{1}$ https://www.esa.int/Safety_Security/Space_Debris/Space_debris_by_the_numbers.
} 
Indeed, the main challenge is to mitigate and reduce the risks posed to space assets, and this has to be achieved by tackling the problem from various directions, each posing different challenges and on different timescales (Bonnal and McKnight, 2017).

\section{SURVEILLANCE AND TRACKING}

Currently, over 22,000 objects (larger than $\sim 10 \mathrm{~cm}$ ) are tracked by Space Surveillance Networks and recorded in their catalog to provide warnings to satellites in the path of these objects and to enable them to perform avoidance maneuvers.

From a technical point of view, here the challenges are the identification, tracking, and cataloging of the centimetersized objects (still large enough to produce catastrophic damage but not included in the current catalogs) and the accurate determination and propagation of the orbits (despite these smaller objects having low orbital stability) to produce reliable conjunction predictions, triggering avoidance maneuvers only when necessary.

Radars have been the preferred ground-based system, in particular to monitor LEO, as they can operate independently day and night as well as in all meteorological conditions. However, most radar telescopes are optimized for astronomical observations rather than debris tracking and so bi-static systems have also been used to improve performance, and some have shown a capability to detect objects down to $1 \mathrm{~cm}$ at $100 \mathrm{~km}$ (Muntoni et al., 2017). Similarly, systems combining laser ranging and passive optical tracking have been demonstrated to achieve good accuracy in determining the position of objects (within $10 \mathrm{~m}$ ).

These capabilities and different organization catalogs have to come together to improve actionable knowledge of the orbital population. All this will underpin the capability to perform an efficient space traffic management system, which will become necessary as the number of satellites increase (Gleason, 2019). However, even the most detailed knowledge of the debris population, although helpful for mitigating the most immediate risks, does not, on its own, solve the main long-term issue, which is to achieve a reduction of the growth and possibly a reversal in the quantity of dangerous space debris.

\section{INTERNATIONAL REGULATIONS}

There are technical and political challenges as well as significant costs involved in implementing effective space debris mitigation (Emanuelli et al., 2014; Yamamoto et al., 2017).

Voluntary debris mitigation guidelines were published in 2002 (and revised in subsequent years ${ }^{2}$ ) by the InterAgency Space Debris Coordination Committee (IADC), an advisory body composed of representatives of the 12 principal governmental space agencies. Subsequently, the nations represented in the U.N. Committee on the Peaceful Uses of Outer Space agreed a voluntary international standard

${ }^{2}$ https://www.unoosa.org/documents/pdf/spacelaw/sd/IADC-2002-01-IADCSpace_Debris-Guidelines-Revision1.pdf. for debris mitigation (ISO 24113) ${ }^{3}$, and several standards and technical reports describing what should be done to address the various issues related to space debris mitigation are available.

However, unfortunately, none of the existing guidelines, regulations, or codes of practice could prevent the testing of anti-satellite weapons, which has been carried out by various nations and has produced significant step increases in the space debris population.

The voluntary nature of the regulatory framework and absence of capability to enforce the regulations pose a challenge that, with the consensus of the various stakeholders, should be addressed.

\section{MITIGATION STRATEGIES}

One of the most well-known guidelines for reducing the growth of the number of pieces of debris in LEO is that objects passing thorough the LEO region should be disposed of (i.e., de-orbited, so that they can burn up when re-entering the Earth's atmosphere and so that any debris that survives and reaches the surface of the planet should not pose an undue risk to people or property, landing in large, uninhabited regions, such as broad ocean areas) within 25 years.

A variety of de-orbit technologies have been considered to ensure that the above-mentioned principle is met (Janhunen, 2010; Forshaw et al., 2015) when the natural orbital decay is insufficient to provide timely end-of-life disposal of a satellite. Techniques have been considered that range from the use of a satellite's own propulsion system to passive devices like deorbit sails (Underwood et al., 2019) to Active Debris Removal (ADR) (Shan et al., 2016). There is a significant overlap between technologies for satellite in-orbit servicing and those for active debris removal, and the commonalities have to be exploited to develop multipurpose technologies and lower the cost of future ADR missions. Some low-cost approaches have been demonstrated in space on artificial targets (Forshaw et al., 2016; Aglietti et al., 2020), but the challenge for the next decade is to perform a real ADR mission and remove one of the large defunct satellites (e.g., Envisat) to reduce the risk of a catastrophic collision in LEO.

\section{PLANETARY PROTECTION}

Last but not least is the challenge to planetary protection from the threat posed by Near Earth Objects that could hit our planet.

Although small objects (meteors) hit our planet on a daily basis without posing any significant threat because they burn through the atmosphere, larger objects do pose a substantial threat, as they can survive contact with the Earth's atmosphere and hit the surface with significant energy. Although the probability of impact by large objects $(100 \mathrm{~s}$ of meters and possibly $\mathrm{kms}$ ) is very low, the craters visible

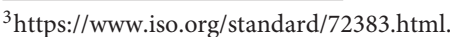


on the Moon and those still visible on Earth (e.g., the 1kilometer-wide Barringer Meteor Crater in Arizona, which was formed $\sim 50,000$ years ago by the impact of a metallic object $\sim 50 \mathrm{~m}$ in diameter) are a reminder that several of these impacts have occurred in the past and, indeed, will happen in the future. As the consequences of such a large impact can be absolutely disastrous, there is a need to develop suitable mitigation strategies.

Through the years, there have been various efforts in this direction. NASA has been active ${ }^{4}$, establishing the Planetary Defense Coordination Office (PDCO) and planning missions like the Double Asteroid Redirection Test (DART). Similarly, ESA has been working on this issue. It proposed the Asteroid Impact Mission (AIM) and then approved the Hera mission to perform key measurements to validate impact and asteroid deflection models. Other space agencies are also working on this topic, and the United Nations have taken useful steps to improve coordination, establishing the International Asteroid Warning Network and the Space Mission Planning Advisory Group (SMPAG). However, a greater level of international coordination and integration of the efforts made by single entities is necessary to produce an effective response.

${ }^{4}$ NASA's Efforts to Identify Near-Earth Objects and Mitigate Hazards IG-14-030 (A-13-016-00) OFFICE OF AUDITS - AUDIT REPORT SEPTEMBER 15, 2014.

\section{REFERENCES}

Aglietti, G. S., Taylor, B., Fellowes, S., Salmon, T., Retat, I., Hall, A., et al. (2020). The active space debris removal mission RemoveDebris. Part 2: in orbit operations. Acta Astronaut. 168, 310-322. doi: 10.1016/j.actaastro.2019.09.001

Bonnal, C., and McKnight, D. S. (2017). IAA Situation Report on Space Debris 2016. Paris: International Academy of Astonautucs.

Emanuelli, M., Prasad, D., and Federico, G. (2014). Conceptualizing an economically, legally, and politically viable active debris removal option. Acta Astronaut. 104, 197-205 doi: 10.1016/j.actaastro.2014.07.035

Forshaw, J. L., Aglietti, G. S., Navarathinam, N., Kadhem, H., Salmon, T., Pisseloup, A., et al. (2016). Removedebris: an in-orbit active debris removal demonstration mission. Acta Astronaut. 127, 448-463. doi: 10.1016/j.actaastro.2016.06.018

Forshaw, J. L., Massimiani, C., Richter, M., Viquerat, A., Simons, E., Duke, R., et al. (2015). Surrey Space Centre: A survey of debris removal research activities, in 66th International Astronautical Congress, International Astronautical Federation, Jerusalem.

Gleason, M. P. (2019). "Establishing space traffic management standards, guidelines and best practices," in First International Orbital Debris Conference, Sugar Land, TX.

Hesse, M., and Hornung, M. (2015). "Space as a critical infrastructure," in Handbook of Space Security, eds K. U. Schrogl, P. Hays, J. Robinson, D. Moura, and C. Giannopapa (New York, NY: Springer).

Janhunen, P. (2010). Electrostatic plasma brake for deorbiting a satellite. J. Propul. Power 26, 370-372. doi: 10.2514/1.47537

Kessler, D. J., and Cour-Palais, B. G. (1978). Collision frequency of artificial satellites: the creation of a debris belt. J. Geophys. Res. Space Phys. 83, 2637-2646. doi: 10.1029/JA083iA06p02637

Muntoni, G., Schirru, L., Pisanu, T., Montisci, G., Valente, G., Gaudiomonte, F., et al. (2017). Space debris detection in low earth orbit with the sardinia radio telescope. Electronics 6:59. doi: 10.3390/electronics 6030059
The challenge is at both levels: political and technical. For the political perspective, there is the need to prepare and agree on the appropriate scenario-dependent plans so that they are ready to be executed in order to produce a timely response and perform the necessary mission. From a technical perspective, besides improvements in detection capabilities and potential impact predictions, the development and testing of methodologies and technologies to deflect a large object (Sanchez Cuartielles et al., 2007) (as this currently seems the most realistic and effective method of intervention) have to progress to the point that they could be deployed with a high level of confidence in their success.

The few topics discussed in this article are by no means an exhaustive list or ranking of the challenges to be overcome in the next few years in the area of space debris, but indeed if we want to continue to exploit space assets to support significant services and protect our environment from events that, although rare, could be catastrophic, addressing the issues described in this article becomes of primary importance.

\section{AUTHOR CONTRIBUTIONS}

The author confirms being the sole contributor of this work and has approved it for publication.
Sanchez Cuartielles, J. P., Colombo, C., Vasile, M., and Radice, G. (2007). "A multi-criteria assessment of deflection methods for dangerous NEOs," in AIP Conference Proceedings, Vol. 886, ed E. Belbruno (Princeton, NJ: AIP Publishing) 886, 317. doi: 10.1063/1.2710065

Shan, M., Guo, J., and Gill, E. (2016). Review and comparison of active space debris capturing and removal methods. Progress Aerospace Sci. 80, 18-32 doi: 10.1016/j.paerosci.2015.11.001

Underwood, C., Viquerat, A., Schenk, M., Taylor, B., Massimiani, C., Duke, R., et al. (2019). InflateSail de-orbit flight demonstration results and follow-on drag-sail applications. Acta Astronaut. 162, 344-358. doi: 10.1016/j.actaastro.2019.05.054

Virgili, B. B., Dolado, J. C., Lewis, H. G., Radtke, J., Krag, H., Revelin, B., et al. (2016). Risk to space sustainability from large constellations of satellites. Acta Astronaut. 126, 154-162. doi: 10.1016/j.actaastro.2016. 03.034

Wang, T. (2010) Analysis of debris from the collision of the cosmos 2251 and the iridium 33 satellites. Sci. Global Sec. 18, 87-118. doi: 10.1080/08929882.2010.493078

Yamamoto, T., Okamoto, H., and Kawamoto, S. (2017). Cost Analysis of Active Debris Removal Scenarios and System Architectures. Darmstadt: 7th European Conference on Space Debris.

Conflict of Interest: The author declares that the research was conducted in the absence of any commercial or financial relationships that could be construed as a potential conflict of interest.

Copyright (C) 2020 Aglietti. This is an open-access article distributed under the terms of the Creative Commons Attribution License (CC BY). The use, distribution or reproduction in other forums is permitted, provided the original author $(s)$ and the copyright owner(s) are credited and that the original publication in this journal is cited, in accordance with accepted academic practice. No use, distribution or reproduction is permitted which does not comply with these terms. 